\title{
Search for single top production at HERA
}

\author{
Lorenzo BELLAGAMBA* \\ Istituto Nazionale di Fisica Nucleare, Via Irnerio 46, I-40126 Bologna, Italy \\ E-mail: lorenzo.bellagamba@bo.infn.it
}

\begin{abstract}
A search for single top production via flavour changing neutral current (FCNC) process has been performed by both the H1 and ZEUS collaborations. H1 exploits the full $e^{ \pm} p$ data sample corresponding to an integrated luminosity of $474 \mathrm{pb}^{-1}$ while ZEUS used 2004-2007 data sample (277 $\mathrm{pb}^{-1}$ ) combined with 1998-2000 data sample $\left(82 \mathrm{pb}^{-1}\right)$. Constraints on the single top production cross section and on the anomalous coupling $\kappa_{t u \gamma}$, mediating the FCNC process, have been derived.
\end{abstract}

European Physical Society Europhysics Conference on High Energy Physics

July 16-22, 2009

Krakow, Poland

${ }^{*}$ Speaker. 


\section{Introduction}

The production of single top quarks in $e p$ collisions at HERA is possible due to the large centre of mass energy, $\sqrt{s}=318 \mathrm{GeV}$. However the dominant process for SM single top production, $e p \rightarrow v t X$, has a predicted cross section of less than $1 \mathrm{fb}$. No sizeable production is hence expected and any observation of top can be attributed to new physics. In several extensions of the SM, single top production can happen via a FCNC process mediated by an effective coupling which allows a $u(c)-t$ transition via a neutral vector boson $\left(\gamma\right.$ or $\left.Z^{0}\right)$. The $\mathrm{H} 1$ and ZEUS collaborations collected, during the HERA operations, an integrated luminosity of $\sim 0.5 \mathrm{fb}^{-1}$ in two running period 1994-2000 (HERA I run) and 2003-2007 (HERA II run). Such data are particularly well suited to investigate FCNC processes involving a coupling $t-u-\gamma$.

\section{Results}

\section{$2.1 \mathrm{H1}$ results}

$\mathrm{H} 1$ has performed the analysis [] investigating the leptonic and hadronic decay channels of the $W$ boson coming from the decay of top quarks. A multivariate discriminant method based on a neural network has been used to differentiate top quark production from background. Figure 1 shows the distribution of the discriminant for the three channels of the $W$ decaying to electron, muon and hadrons. Black dots are data, the full line histograms are the SM predictions and the dashed lines correspond to the single top signal (ANOTOP [2]) with top mass set to $M_{t o p}=175$ $\mathrm{GeV}$. The normalisation of the signal is arbitrary. The main SM background is due to the production of single $W$ boson [3]. Other background sources are neutral current (NC), charged current (CC), photoproduction and lepton pair production. The search in the electron and muon channels is based on the selection described in [他, while the search in the hadron channel is based on selecting events with at least three jets in the pseudorapidity range $-0.5<\eta^{\text {jet }}<2.5$ ordered by the magnitude of the transverse momentum and passing the cuts $P_{T}^{\text {jet } 1}>40 \mathrm{GeV}, P_{T}^{\text {jet } 2}>30 \mathrm{GeV}$ and $P_{T}^{\text {jet } 3}>15$ $\mathrm{GeV}$. In addition, one of the jet pairings must yield an invariant mass between 65 and $95 \mathrm{GeV}$, a window around the $W$ nominal mass. Since no significant excess is seen, limits on the single top production have been set using the method of fractional counting which takes into account efficiencies, statistical and systematic uncertainties. Combining all channels, an upper limit on the cross section is obtained at 95\% C.L.: $\sigma(e p \rightarrow e t X, \sqrt{s}=318 G e V)<0.25 \mathrm{pb}$. The limit
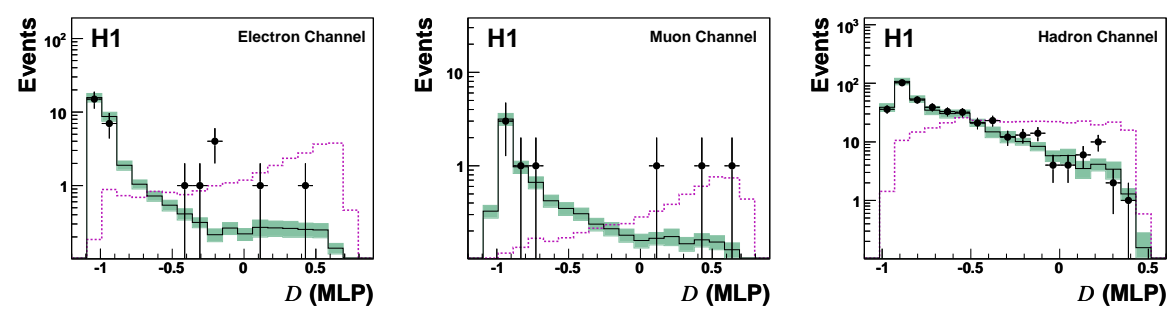

Figure 1: Plot of the discriminant in the electron (left), muon (middle), hadron (right) channels for the H1 analysis. 
on the cross section is converted into limit on the anomalous FCNC coupling $\kappa_{t u \gamma}$ at $95 \%$ C.L.: $\kappa_{t u \gamma}<0.16-0.18$ for the top mass range $M_{t o p}=170-175 \mathrm{GeV}$.

\subsection{ZEUS results}

The ZEUS analysis have been performed using data collected in 2004-2007 corresponding to an integrated luminosity of $277 \mathrm{pb}^{-1}$. The search has been performed in the electron and muon channels starting with a generic selection requiring an isolated high- $P_{T}$ lepton $\left(P_{T, e l}>10 \mathrm{GeV}\right.$, $P_{T, \mu}>8 \mathrm{GeV}$ ) and large missing transverse momentum, $P_{T, \text { miss }}>10 \mathrm{GeV}$ and $P_{T, \text { miss }}>12 \mathrm{GeV}$ for the muon and electron channels, respectively. The SM $W$ production has been modelled using EPVEC [3]; other background processes considered are: lepton pair production, neutral current DIS (NC), charged current DIS (CC) and photoproduction. Single top sample has been generated with CompHEP [5] and Pythia 6.14 [6] for the parton shower, hadronization and particle decays. Figure 2 shows the preselection plots in the electron (left plot) and muon (right plot) channels for several relevant variables. The main sources of background are NC DIS and di-muon production for the electron and muon channels, respectively. Since no large discrepancy is observed at high hadronic $P_{T}$, where the single-top production is expected to contribute, limits were set for this process. A cut on the hadronic $P_{T}>40 \mathrm{GeV}$ has been set and then the cuts on acoplanarity and missing $P_{T}$ were optimised. Table 1 shows the comparison between data and SM expectations after the final selection for the different samples and decay channels. Such results have been used to

Single Top Selection

\begin{tabular}{|ccccc|}
\hline & $N_{\text {obs }}$ & $N_{\text {pred }}$ & $W \%$ & Efficiency \\
\hline Electron Channel 04-05 $e^{-} p$ & 0 & $2.1 \pm 0.6$ & 38 & 0.033 \\
Muon Channel 04-05 $e^{-} p$ & 1 & $1.5 \pm 0.4$ & 47 & 0.026 \\
\hline Electron Channel 06-07 $e^{+} p$ & 0 & $0.9 \pm 0.3$ & 78 & 0.033 \\
Muon Channel 06-07 $e^{+} p$ & 1 & $1.4 \pm 0.4$ & 50 & 0.026 \\
\hline
\end{tabular}

Table 1: Table of events passing the ZEUS final selection cuts. The errors in the MonteCarlo have been obtained adding in quadrature systematic and statistical contributions which are of the same order.

constraint single top production by means of a bayesian approach assuming a constant prior on the signal cross section. The following upper limit on the cross section has been obtained: $\sigma<0.23$ $\mathrm{pb}(95 \%$ C.L.). The limit on the cross section has been converted into a limit on the coupling $\kappa_{t u \gamma}$ using the signal cross section evaluated by CompHEP: $\kappa_{t u \gamma}<0.17$ with $M_{t o p}=171.2 \pm 2.1$ (PDG value). Varying the top mass between its uncertainty, $\kappa_{t u \gamma}$ varies between 0.16 and 0.18 . The limit is similar to the one obtained by ZEUS with 1994-2000 data [7]. The present result has been combined with the limit, obtained using the HERA I data sample with $\sqrt{s}=318 \mathrm{GeV}$, for a total integrated luminosity of $359 \mathrm{pb}^{-1}$. The combined cross section and $\kappa_{t u \gamma}$ limits are $\sigma<0.13 \mathrm{pb}$ and $\kappa_{t u \gamma}<0.13$, respectively.

\section{Conclusions}

Single top production via FCNC has been searched for with the H1 and ZEUS detectors at HERA. The results have been used to set limits on the cross section and on the coupling $\kappa_{t u \gamma}$. 

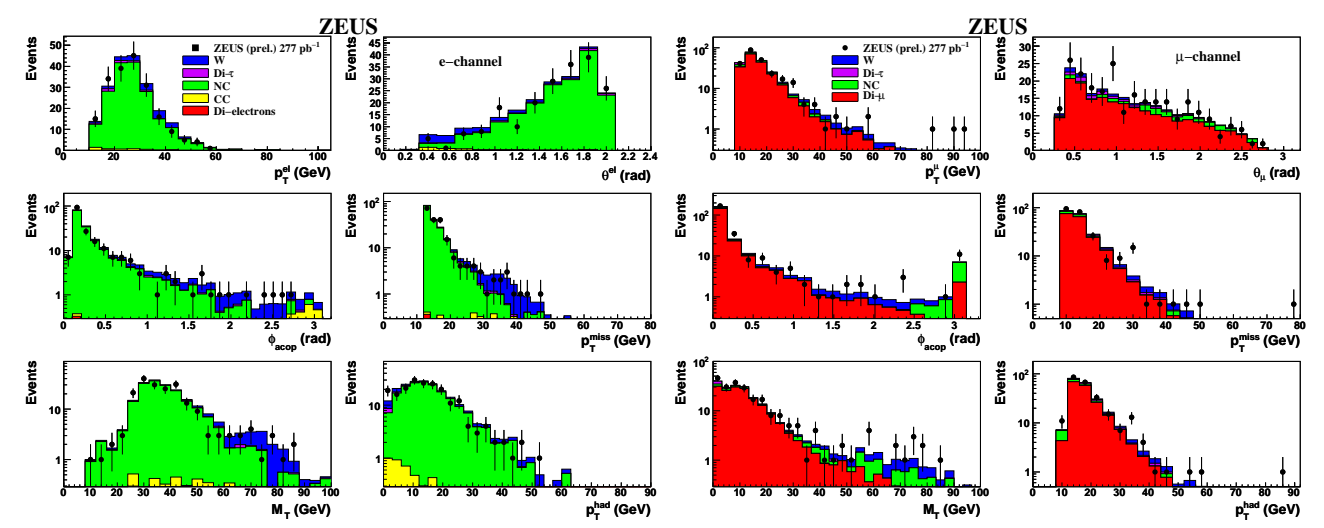

Figure 2: Preselection plots in the electron (left) and muon (right) channel for the ZEUS analysis. For each channel the following variables are shown: $P_{t}$ and $\theta$ of the lepton, acoplanarity, missing $P_{t}$, transverse mass and hadronic $P_{t}$.



Figure 3: Constraints in the $\kappa_{t u \gamma}-V_{t u z}$ plane for the different colliders.

Figure 3 shows the constraints from different colliders in the plane of the couplings $\kappa_{t u \gamma}$ and $V_{t u Z}$. HERA limits are the most stringent for the $\kappa_{t u \gamma}$ coupling.

\section{References}

[1] H1 Coll., DESY 09-050 (2009).

[2] A.Aktas et al. [H1 Coll.], Eur.Phys.J. C33 9 (2004).

[3] U.Baur,J.A.Vermaseren and D.Zeppenfeld, Nucl.Phys. B375 3 (1992).

[4] F.D.Aron et al. [H1 Coll.], arXiv:hep-ex/0901.0488 (2009).

[5] E.Boos et al., Nucl.Inst.Meth $\mathbf{A 5 3 4} 250$ (2004).

[6] T.Sjöstrand, P. Eden, C. Friberg, L. Lönnblad, G. Miu, S. Mrenna and E. Norrbin, Comp. Phys. Comm. 135238 (2001)

[7] ZEUS Coll., Phys.Lett. B559 153-170 (2003). 